\title{
VARIATIONS IN THE PULMONARY CAPILLARY BLOOD VOLUME AND MEMBRANE DIFFUSION COMPONENT IN HEALTH AND DISEASE *
}

\author{
By D. V. BATES, C. J. VARVIS, R. E. DONEVAN AND R. V. CHRISTIE \\ (From the Joint Cardio-Respiratory Service, Royal Victoria Hospital and Montreal Children's \\ Hospital, McGill University, Montreal, Canada)
}

(Submitted for publication February 17, 1960; accepted May 13, 1960)

Forster, Roughton and their colleagues $(1,2)$ have reported a method of subdividing the components of the pulmonary diffusing capacity by measurement of the apparent diffusing capacity at different alveolar oxygen tensions. This development followed preparatory work by the same authors $(3,4)$ in which the kinetic factors involved in $\mathrm{CO}$ and hemoglobin combination were reported. The principles underlying the measurements have been fully discussed by Forster in a recent review (5).

Lewis, Lin, Noe and Komisaruk (6) reported on the measurement of pulmonary capillary blood volume in normal subjects, using the single breath method of determining the pulmonary diffusing capacity. They found average values of $65 \mathrm{ml}$ for the pulmonary capillary blood volume at rest and $98 \mathrm{ml} \mathrm{CO}$ per minute per $\mathrm{mm} \mathrm{Hg}$ for the membrane diffusion component. In four subjects both of these quantities increased somewhat during exercise. They did not report any results on patients. McNeill, Rankin and Forster (7), using a similar technique of measuring the pulmonary diffusing capacity by a single breath method, found similar values at rest for the pulmonary capillary blood volume, but a lower value for the membrane component, which was about $63.5 \mathrm{ml}$ $\mathrm{CO}$ per minute per $\mathrm{mm} \mathrm{Hg}$ in normal subjects at rest. These authors reported the results of this measurement in five patients with various types of pulmonary fibrosis, two with pulmonary hypertension, two with pulmonary congestion, and three with chronic obstructive emphysema. The most significant findings were the decrease in membrane diffusion component in patients with pulmonary fibrosis, and the increase generally found in pulmonary capillary blood volume in patients with increased pressure in the lesser circulation.

The present study reports the measurement of

* Supported by a grant from the National Research Council of Canada. these two components of the pulmonary diffusing capacity by a steady state method during exercise in 14 normal subjects and in a group of 22 patients. This work was undertaken to study the reliability of the results obtainable with this technique, and to explore its usefulness in investigative work.

\section{METHODS}

The apparatus used in this work has recently been described in full (8). A steady state measurement of diffusing capacity (DL) is made, the mean alveolar CO tension being calculated from an assumed value of respiratory dead space. Technical details and a study of the errors of this method have been presented previously (8). The determination of pulmonary capillary blood volume $\left(V_{c}\right)$ and membrane diffusion component (DM) involves the measurement of the pulmonary diffusing capacity at two different oxygen tensions. Each experiment whether on a normal subject or a patient consisted in the measurement of the diffusing capacity on either 60 or 100 per cent oxygen, followed by its determination on air. All estimates were made during moderate or light exercise.

In a typical determination, the subject attained a steady state on the exercise treadmill for 5 minutes, and was then switched into either 60 or 100 per cent oxygen for a further period of about 5 minutes. At the end of this time, without interruption of the exercise, he was connected to the inspiratory bag which contained the same oxygen concentration as he had been breathing, with the addition of approximately 0.20 per cent CO. The inspired and expired gases were monitored in the manner described previously, and a careful note was made of the total time during which the subject breathed $\mathrm{CO}$. The subject then rested on a chair, and during this time a sample of venous blood was drawn for the measurement of carboxyhemoglobin ( $\mathrm{COHb}$ ) by the technique of Lawther and Apthorp (9). With the treadmill in the same position and at the same speed, the subject then resumed his exercise, the sequence of events being timed as before, but on this occasion he was switched into a mixture of air containing about 0.12 per cent $\mathrm{CO}$. Following the determinations of the inspired and mixed expired $\mathrm{CO}$ concentrations, the oxygen uptake was calculated using the monitored mixed expired $\mathrm{CO}_{2}$ and oxygen in the manner previously described (8). The total experiment 
with the two measurements of diffusing capacity took about 25 minutes. Values for $D_{L}$ at each oxygen tension were not "pooled"; each experiment consisted in the double determination of DL followed by the calculation of $V_{c}$ and $D_{M}$ values from these two points. This procedure was followed so that the standard deviation of repeat determinations would provide an indication of the likely error of a single determination of $V_{c}$ and $D_{M}$ based on one determination of $D_{L}$ at each oxygen tension. A higher inspired CO concentration was used during the 100 per cent oxygen determination to lower the proportionate effect of "back pressure" of $\mathrm{CO}$ in relation to alveolar CO concentration.

\section{CALCULATIONS}

The pulmonary diffusing capacity was calculated from the $\mathrm{DCO}_{1 \mathrm{~A}}$ as previously described (8) except that the $\mathrm{COHb}$ correction was slightly modified. Early experience with the method showed that the calculated values of $V_{c}$ and $D_{M}$ were very sensitive to this correction, and it was found necessary to calculate the mean $\mathrm{COHb}$ during the exercise run; rather than to use the $\mathrm{COHb}$ level measured at the end of exercise as the pertinent value. This change alters the calculated DL by not more than one unit, but this is sufficient to affect the calculated $V_{c}$.

The corrected DL was calculated as previously described by Linderholm's method (10). The circulating blood volume of the subject was calculated from the formulas:
$32.18 \mathrm{cc}$ per $\mathrm{lb}$ in males and $32.14 \mathrm{cc}$ per $\mathrm{lb}$ in females.

The alveolar oxygen tension $\left(\mathrm{PAO}_{2}\right)$ was calculated from the Bohr alveolar gas equation using a mixed expired oxygen percentage of $\left(100-\mathrm{FECO}_{2}\right)$ or $(60-$ $\mathrm{FE}_{\mathrm{CO}_{2}}$ ) depending on which oxygen mixture had been used. During the air diffusing capacity determination the measured mixed expired oxygen concentration was used in this calculation. Previous work from this laboratory (8) has supported the view of Asmussen and Neilsen (11) that the alveolar oxygen so calculated during exercise in normal subjects is reasonably accurate. The mean capillary oxygen tension $\left(\overline{\mathrm{P}}_{\mathrm{C}_{2}}\right)$ was then calculated by the method described by Linderholm (10). A " $\lambda$ value" of $2.5(2,3,5)$. was assumed for all subjects. The function $1 / \theta$ was calculated from the formula,

$$
1 / \theta=\left[\left(0.0057 \times \overline{\mathrm{P}_{\mathrm{C}_{2}}}\right)\right]+0.75,
$$

which was derived from Figure 1 (2).

The pulmonary midcapacity during exercise was calculated by adding half the tidal volume to the measured resting functional residual capacity (FRC), a technique shown to give a reasonably accurate estimate of this figure during exercise (8).

The $\mathrm{V}_{\mathrm{c}}$ and the $\mathrm{D}_{\mathrm{M}}$ were then calculated by solving the equation $1 / \mathrm{DL}_{\mathrm{LO}}=1 / \theta \mathrm{V}_{\mathrm{c}}+1 / \mathrm{DM}_{\mathrm{M}}$ for the two determinations of pulmonary diffusing capacity at the two oxygen tensions. It will be appreciated that this calculation necessarily involves the assumption that the switch

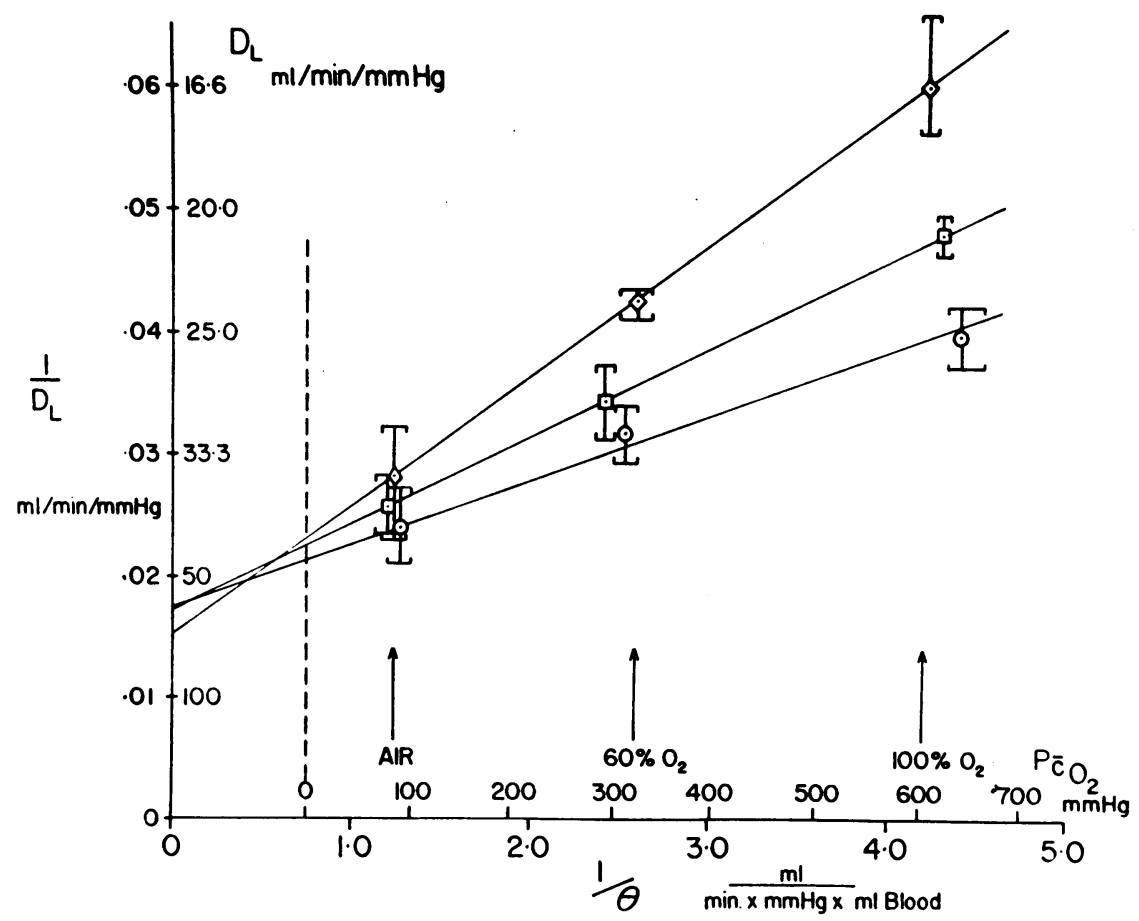

Fig. 1. Relationship between 1/Dl and mean alveolar capillary oxygen TENSION $\left(\overline{\mathrm{P}}_{\mathrm{CO}_{2}}\right)$ IN SUBjects RED $\odot, \mathrm{CJV} \odot$, and DVB $\odot$. The lines about the mean values represent the actual ranges of the observation of $\mathrm{DL}_{L}$ and $\overline{\mathrm{P}} \mathrm{C}_{\mathrm{O}_{2}}$ in these subjects, all of whom were exercising at $3 \mathrm{mph}$ on a flat surface. 
from air to oxygen has not changed the pulmonary capillary bed significantly, nor altered the nature or area of the pulmonary membrane.

Very accurate determinations of $D_{L}$ are required if variability in the calculated pulmonary capillary blood volumes and membrane components is to be avoided. An important precaution is the avoidance of contaminated $\mathrm{CO}$ mixtures as mentioned previously (8), and accuracy in the determination of $\mathrm{COHb}$. The correction due to this factor becomes considerable if a heavy smoker is studied, and is more significant when he is breathing oxygen than air. We have found that the calculated back tension of $\mathrm{CO}$ must not be allowed to exceed more than 15 per cent of the alveolar CO pressure if considerable errors in the determined value of $\mathrm{V}_{\mathbf{c}}$ are to be avoided. When heavy smokers are studied, the inspired $\mathrm{CO}$ percentage may have to be increased to 0.30 per cent during the 100 per cent oxygen determination to keep the calculated back $\mathrm{CO}$ tension less than 15 per cent of the alveolar $\mathrm{CO}$ tension.

\section{RESULTS}

Normal subjects. The results are shown in Tables I through VIII, and in Figure 1. In Table I are shown the physical characteristics of the 14 normal subjects studied, together with the results of resting pulmonary function tests. The ages of these subjects varied from 24 to 46 . The resting pulmonary function tests were all within predicted limits. In Table II are shown the results of determinations of pulmonary capillary blood volumes and membrane diffusion components in four of these subjects. At a speed of 3 mph with the treadmill flat, a total of 20 determinations was made on these four subjects, all on different days, and four observations were made on Subject DVB at $3 \mathrm{mph}$ on a 10 per cent treadmill grade. The ranges of variation together with the mean values are shown in this table and below each column are the standard deviations of the observations. The extreme right-hand column of this table shows the number of estimations done on 100 or 60 per cent oxygen on each individual. The most variable results were in the determination of $V_{c}$ in Subject RED. One determination was double another at approximately the same oxygen uptake and this led to the finding of a high standard deviation for the five observations recorded. If this single result be omitted, the standard deviations are less than \pm 12.5 for the determination of $\mathrm{V}_{\mathrm{c}}$ on these four subjects. It is clear from the data that there are significant differences in the mean values of $V_{c}$ between different normal subjects, those, for instance, in LSB being uniformly and significantly lower than those in CJV or RED. There is less variability and rather less variation between individuals in the measured DM, and the differences in this component disappear when it is related to lung volume (DM/MC). The $\mathrm{V}_{\mathbf{c}}$ is still significantly different after this correction, however $\left(V_{c} / M C\right)$. In Subject DVB, an increase in exercise resulted in a significant increase in the over-all DL on air (DL/air), and in $\mathrm{V}_{\mathrm{c}}$, but there was no change in DM. This suggests that the increased DL on exercise is mainly accounted for by an increase in $\mathrm{V}_{\mathfrak{c}}$, though this conclusion might not necessarily be applicable to the transition from rest to exercise.

In Table III, the subjects who are below 35

TABLE I

Physical data and results of resting pulmonary function tests in normal subjects*

\begin{tabular}{|c|c|c|c|c|c|c|c|c|c|}
\hline No. & Subject & Sex & Age & $\mathrm{Ht}$ & $\mathrm{Wt}$ & VC & FRC & ME\% & MMFR \\
\hline & & & & in & $l b s$ & $L$ & $L$ & & $L / \mathrm{sec}$ \\
\hline 1 & RED & $\mathbf{M}$ & 30 & $70 \frac{1}{2}$ & 165 & 4.97 & 3.18 & 67 & 3.92 \\
\hline 2 & CJV & M & 29 & $71 \frac{1}{2}$ & 195 & 5.04 & 2.92 & 78 & 4.60 \\
\hline 3 & $F M$ & M & 28 & 74 & 205 & 5.77 & 3.77 & 60 & 6.80 \\
\hline 4 & GW & M & 34 & $69 \frac{1}{2}$ & 168 & 4.91 & 3.54 & 66 & 5.90 \\
\hline 5 & DP & M & 24 & $72 \frac{1}{2}$ & 182 & 5.04 & 3.60 & 71 & 5.00 \\
\hline 6 & $\mathrm{RF}$ & M & 25 & 65 & 137 & 4.40 & 2.28 & 71 & 6.00 \\
\hline 7 & $\mathrm{JE}$ & M & 31 & 71 & 186 & $(4.10)$ & $(3.20)$ & & \\
\hline 8 & DVB & M & 37 & 70 & 180 & 3.33 & 2.75 & 92 & 3.41 \\
\hline 9 & LSB & M & 41 & $66 \frac{1}{2}$ & 168 & 3.00 & 3.26 & 58 & 3.50 \\
\hline 10 & REF & M & 39 & 74 & 165 & $(4.40)$ & $(3.50)$ & & \\
\hline 11 & WF & M & 44 & 68 & 163 & 4.45 & 2.91 & 52 & 2.80 \\
\hline 12 & JAP & $\mathrm{M}$ & 42 & 75 & 193 & 4.00 & 3.21 & 58 & 3.90 \\
\hline 13 & REGP & M & 46 & $66 \frac{1}{2}$ & 173 & 3.58 & 1.72 & 53 & 3.00 \\
\hline 14 & JB & M & 45 & 72 & $211 \frac{1}{2}$ & 4.95 & 3.02 & 91 & 7.00 \\
\hline
\end{tabular}

*VC = vital capacity $; \mathrm{FRC}=$ functional residual capacity; $\mathrm{ME} \%=$ closed circuit helium mixing index; MMFR $=$ maximal midexpiratory flow rate. Results shown in parentheses have been predicted from the subject's height. 
TABLE II

Results of repeated estimates of pulmonary capillary blood volume $\left(V_{c}\right)$ and membrane diffusion component $\left(D_{M}\right)$ in four normal subjects*

\begin{tabular}{|c|c|c|c|c|c|c|c|c|c|c|c|c|}
\hline Subject & Rate & Grade & $\underset{\text { vol }}{\text { Minute }}$ & $\mathrm{Vo}_{2}$ & 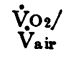 & $\begin{array}{l}\text { DLco } \\
\text { (air) }\end{array}$ & $\mathrm{V}_{0}$ & DM & $\begin{array}{l}\text { DLoo/ } \\
\text { MC }\end{array}$ & $\begin{array}{l}\mathrm{V}_{\mathrm{c}} \mathrm{l} \\
\mathrm{MC}\end{array}$ & $\begin{array}{l}\text { DM/ } \\
\text { MC }\end{array}$ & $\begin{array}{c}\text { No. of } \\
\text { estimations }\end{array}$ \\
\hline DVB & $m p h$ & & $l / \min$ & $l / \min$ & & & $m l$ & & & & & \\
\hline $\begin{array}{l}\text { Mean } \dagger \\
\text { SD } \pm\end{array}$ & 3 & $10 \%$ & $\begin{array}{r}43.6 \\
49.2 \\
53.5 \\
3.6\end{array}$ & $\begin{array}{l}1.68 \\
1.90 \\
2.22 \\
0.19\end{array}$ & $\begin{array}{l}3.42 \\
3.86 \\
4.17 \\
0.31\end{array}$ & $\begin{array}{l}39.3 \\
41.0 \\
43.9 \\
1.34\end{array}$ & $\begin{array}{l}122 \\
131 \\
135 \\
5.25\end{array}$ & $\begin{array}{l}62.0 \\
64.2 \\
68.0 \\
2.36\end{array}$ & $\begin{array}{c}9.62 \\
10.1 \\
11.0 \\
0.53\end{array}$ & $\begin{array}{c}29.6 \\
32.3 \\
33.9 \\
1.64\end{array}$ & $\begin{array}{r}15.3 \\
15.8 \\
16.4 \\
0.5\end{array}$ & $\begin{array}{r}100 \% \mathrm{O}_{2} \times 3 \\
60 \% \mathrm{O}_{2} \times 1 \\
\text { Total }=4\end{array}$ \\
\hline $\begin{array}{l}\text { Mean } \\
\text { SD } \pm\end{array}$ & 3 & Flat & $\begin{array}{l}25.7 \\
28.4 \\
30.2 \\
1.45\end{array}$ & $\begin{array}{l}1.10 \\
1.21 \\
1.29 \\
0.07\end{array}$ & $\begin{array}{l}3.85 \\
4.26 \\
4.63 \\
0.26\end{array}$ & $\begin{array}{c}32.5 \\
35.3 \\
38.6 \\
2.34\end{array}$ & $\begin{array}{c}81 \\
96 \\
109 \\
11.1\end{array}$ & $\begin{array}{l}52.2 \\
66.5 \\
87.0 \\
11.4\end{array}$ & $\begin{array}{l}8.6 \\
9.2 \\
9.91 \\
0.40\end{array}$ & $\begin{array}{c}20.1 \\
25.3 \\
30.3 \\
3.39\end{array}$ & $\begin{array}{r}14.5 \\
17.4 \\
21.5 \\
2.5\end{array}$ & $\begin{array}{r}100 \% \mathrm{O}_{2} \times 3 \\
60 \% \\
\text { Total } \mathrm{O}_{2} \times 3 \\
=\end{array}$ \\
\hline $\begin{array}{l}\text { RED } \\
\text { Mean } \\
\text { SD } \pm \\
\text { CJV }\end{array}$ & 3 & Flat & $\begin{array}{l}22.8 \\
25.3 \\
27.4 \\
1.53\end{array}$ & $\begin{array}{l}1.13 \\
1.21 \\
1.33 \\
0.08\end{array}$ & $\begin{array}{l}5.37 \\
4.79 \\
4.17 \\
0.43\end{array}$ & $\begin{array}{r}35.3 \\
38.8 \\
44.0 \\
3.0\end{array}$ & $\begin{array}{l}122 \\
163 \\
261 \\
50.4\end{array}$ & $\begin{array}{l}45.3 \\
58.2 \\
75.7 \\
11.8\end{array}$ & $\begin{array}{r}9.21 \\
10.0 \\
11.10 \\
0.65\end{array}$ & $\begin{array}{l}31.3 \\
42.0 \\
67.5 \\
13.2\end{array}$ & $\begin{array}{l}11.9 \\
15.0 \\
19.1 \\
2.84\end{array}$ & $\begin{array}{r}100 \% \mathrm{O}_{2} \times 2 \\
60 \% \mathrm{O}_{2} \times 3 \\
\text { Total }=5\end{array}$ \\
\hline $\begin{array}{l}\text { Mean } \\
\text { SD } \pm \\
\text { LSB }\end{array}$ & 3 & Flat & $\begin{array}{c}28.1 \\
31.7 \\
39.1 \\
3.82\end{array}$ & $\begin{array}{l}1.21 \\
1.27 \\
1.38 \\
0.06\end{array}$ & $\begin{array}{l}3.17 \\
4.01 \\
4.42 \\
0.46\end{array}$ & $\begin{array}{r}37.0 \\
41.5 \\
46.8 \\
4.2\end{array}$ & $\begin{array}{c}182 \\
200 \\
214 \\
12.5\end{array}$ & $\begin{array}{r}47.0 \\
56.8 \\
67.2 \\
8.1\end{array}$ & $\begin{array}{c}9.05 \\
10.3 \\
11.5 \\
1.04\end{array}$ & $\begin{array}{r}46.8 \\
49.4 \\
52.7 \\
2.2\end{array}$ & $\begin{array}{r}11.6 \\
14.0 \\
16.5 \\
2.1\end{array}$ & $\begin{array}{r}100 \% \mathrm{O}_{2} \\
60 \% \\
\text { Total } \\
\mathrm{O}_{2}\end{array} \times \begin{array}{l}3 \\
=\end{array}$ \\
\hline $\begin{array}{l}\text { Mean } \\
\text { SD } \pm\end{array}$ & 3 & Flat & $\begin{array}{r}21.1 \\
23.7 \\
24.9 \\
1.21\end{array}$ & $\begin{array}{l}0.98 \\
1.04 \\
1.14 \\
0.06\end{array}$ & $\begin{array}{l}4.02 \\
4.39 \\
4.80 \\
0.30\end{array}$ & $\begin{array}{l}20.2 \\
22.1 \\
23.9 \\
\cdot 1.6\end{array}$ & $\begin{array}{l}58.0 \\
68 \\
80.2 \\
8.45\end{array}$ & $\begin{array}{l}38.5 \\
40.2 \\
43.1 \\
1.55\end{array}$ & $\begin{array}{l}6.85 \\
7.5 \\
7.96 \\
0.43\end{array}$ & $\begin{array}{l}19.7 \\
22.9 \\
26.6 \\
24.8\end{array}$ & $\begin{array}{r}13.1 \\
13.9 \\
14.4 \\
5.2\end{array}$ & $\begin{array}{r}100 \% \mathrm{O}_{2} \times 2 \\
60 \% \mathrm{O} \\
\text { Total } \underset{\mathrm{O}_{2}}{=} \times \frac{2}{4}\end{array}$ \\
\hline
\end{tabular}

* Columns 9,10 and 11 show the values given in the preceding three columns divided by the exercise midcapacity in liters. The final column shows the number of estimates done on air and 100 per cent oxygen, or on air and 60 per cent oxygen on each subject. Vंo ${ }_{2}=o_{0 x y g e n}$ consumption; $\dot{\mathrm{VO}}_{2} / \dot{\mathrm{V}}_{\mathrm{air}}=$ oxygen consumption per liter ventilation; $\mathrm{DL}_{\mathrm{CO}}=$ diffusing capacity of the lung for $\mathrm{CO} ; \mathrm{MC}=$ ventilated lung volume.

$\dagger$ Figures above and below the mean value indicate the range of observations.

years of age have been grouped together for convenience so that their data may be compared to those of the normal subjects over 35 years of age shown in Table IV. The difference in $\mathrm{DL}_{\mathrm{co}}$ between these two groups, 37.4 in the younger and 30.5 in the older, is significant at the 2 per cent level, confirming work previously reported from this laboratory (8). However, the differences of
$20 \mathrm{ml}$ in $\mathrm{V}_{\mathrm{c}}$ and 7.9 in DM are not statistically significant in these small groups of subjects. More work will be required before the cause of the lowered DL with age can be confidently attributed either to changes in $V_{c}$ or DM or both.

Validation of the theoretical considerations underlying this technique requires that the relationship between $1 / \mathrm{DL}$ and $1 / \theta$ should be linear.

TABLE III

Determinations of pulmonary capillary blood volume and membrane diffusion components on seven normal subjects below the age of $35^{*}$

\begin{tabular}{|c|c|c|c|c|c|c|c|c|c|c|c|}
\hline No. & Subject & $\underset{\text { vol }}{\text { Minute }}$ & $\dot{\mathrm{VO}}_{2}$ & $\begin{array}{l}\dot{V}_{\text {Vog/ }} \\
\dot{V}_{\text {air }}\end{array}$ & $\begin{array}{l}\text { Dloo } \\
\text { (air) }\end{array}$ & $\begin{array}{l}\text { DLoo } \\
\left(\mathrm{O}_{2}\right)\end{array}$ & $V_{c}$ & DM & $\begin{array}{l}\text { DLCo/ } \\
\text { MC }\end{array}$ & $\begin{array}{l}\mathrm{V}_{\mathrm{c}} / \\
\mathrm{MC}\end{array}$ & $\begin{array}{l}\text { DM/ } \\
\text { MC }\end{array}$ \\
\hline \multirow{4}{*}{$\begin{array}{l}1 \\
2 \\
3 \\
4 \\
5 \\
6 \\
7\end{array}$} & & $L$ & $L / \min$ & & & & $m l$ & $\underset{m i n /}{\operatorname{mi} C O /}$ & & & \\
\hline & $\begin{array}{l}\text { RED } \\
\text { CJV } \\
\text { FM } \\
\text { GW } \\
\text { DP } \\
\text { RF } \\
\text { JE }\end{array}$ & $\begin{array}{l}25.3 \\
31.7 \\
27.8 \\
31.9 \\
22.6 \\
25.9 \\
36.2\end{array}$ & $\begin{array}{l}1.21 \\
1.27 \\
1.42 \\
1.11 \\
1.10 \\
1.01 \\
1.89\end{array}$ & $\begin{array}{l}4.79 \\
4.01 \\
4.80 \\
3.38 \\
4.70 \\
3.41 \\
4.31\end{array}$ & $\begin{array}{l}38.8 \\
41.4 \\
45.3 \\
39.7 \\
30.9 \\
29.0 \\
37.0\end{array}$ & $\begin{array}{l}21.4 \\
25.2 \\
26.7 \\
22.2 \\
18.9 \\
15.9 \\
18.1\end{array}$ & $\begin{array}{l}163 \\
200 \\
199 \\
152 \\
150 \\
104 \\
105\end{array}$ & $\begin{array}{l}58.2 \\
56.8 \\
62.3 \\
60.2 \\
41.2 \\
45.0 \\
60.0\end{array}$ & $\begin{array}{r}10.0 \\
10.3 \\
9.3 \\
9.1 \\
6.9 \\
8.5 \\
8.8\end{array}$ & $\begin{array}{l}42.0 \\
49.5 \\
40.5 \\
34.7 \\
33.6 \\
30.2 \\
24.9\end{array}$ & $\begin{array}{r}15.0 \\
14.1 \\
12.7 \\
13.7 \\
9.2 \\
13.1 \\
14.3\end{array}$ \\
\hline & Mean $\dagger$ & $\begin{array}{l}22.6 \\
28.8 \\
36.2\end{array}$ & $\begin{array}{l}1.01 \\
1.29 \\
1.89\end{array}$ & $\begin{array}{l}3.38 \\
4.20 \\
4.79\end{array}$ & $\begin{array}{l}29.0 \\
37.4 \\
45.3\end{array}$ & $\begin{array}{l}15.9 \\
21.2 \\
26.7\end{array}$ & $\begin{array}{l}104 \\
153 \\
200\end{array}$ & $\begin{array}{l}41.2 \\
54.8 \\
62.3\end{array}$ & $\begin{array}{r}6.9 \\
9.0 \\
10.3\end{array}$ & $\begin{array}{l}24.9 \\
36.5 \\
49.5\end{array}$ & $\begin{array}{r}9.2 \\
13.2 \\
15.0\end{array}$ \\
\hline & $\mathrm{SD}$ & \pm 4.35 & \pm 0.27 & \pm 0.57 & \pm 5.32 & \pm 3.12 & \pm 36.1 & \pm 7.7 & \pm 1.0 & \pm 7.5 & \pm 1.76 \\
\hline
\end{tabular}

* All estimates done on exercise at $3 \mathrm{mph}$ on a flat surface. Column headings are as in Table II.

$\dagger$ Figures above and below the mean value indicate the range of observations. 
TABLE IV

Determinations of pulmonary capillary blood and membrane diffusion components in the seven normal subjects above the age of $35^{*}$

\begin{tabular}{|c|c|c|c|c|c|c|c|c|c|c|c|}
\hline No. & Subject & $\underset{\text { vol }}{\text { Minute }}$ & $\dot{\mathrm{V}}_{2}$ & 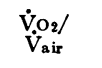 & $\begin{array}{l}\text { DLco } \\
\text { (air) }\end{array}$ & $\begin{array}{l}\text { DLco } \\
\left(\mathrm{O}_{2}\right)\end{array}$ & $V_{0}$ & DM & $\begin{array}{l}\text { DLoo/ } \\
\text { MC }\end{array}$ & $\begin{array}{l}\mathrm{V}_{\mathrm{c}} / \\
\mathrm{MC}\end{array}$ & $\begin{array}{l}\text { DM/ } \\
\text { MC }\end{array}$ \\
\hline \multirow{12}{*}{$\begin{array}{r}8 \\
9 \\
10 \\
11 \\
12 \\
13 \\
14\end{array}$} & & $L$ & $L / \min$ & & & & $m l$ & 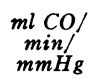 & & & \\
\hline & DVB & 28.4 & 1.21 & 4.26 & 35.2 & 16.7 & 96 & 66.5 & 9.2 & 25.3 & 17.4 \\
\hline & LSB & 23.7 & 1.04 & 4.39 & 22.1 & 11.9 & 68 & 40.2 & 7.4 & 22.9 & 13.7 \\
\hline & REF & 26.8 & 1.30 & 4.21 & 35.3 & 22.0 & 180 & 46.7 & 8.1 & 41.2 & 10.6 \\
\hline & WF & 27.6 & 1.03 & 3.51 & 32.6 & 17.4 & 114 & 51.7 & 6.9 & 24.4 & 11.1 \\
\hline & JAP & 25.8 & 1.18 & 4.11 & 30.2 & 19.8 & 176 & 46.0 & 7.9 & 38.2 & 10.0 \\
\hline & REGP & 29.5 & 1.17 & 3.83 & 22.5 & 12.5 & 83 & 33.1 & 9.1 & 33.4 & 13.4 \\
\hline & $\mathrm{JB}$ & 24.7 & 1.23 & 4.65 & 35.5 & 23.4 & 214 & 44.2 & 9.5 & 56.9 & 11.8 \\
\hline & & 23.7 & 1.03 & 3.51 & 22.1 & 11.9 & 68 & 33.1 & 7.4 & 22.9 & 10.0 \\
\hline & Mean $\dagger$ & 26.6 & 1.17 & 4.14 & 30.5 & 17.6 & 133 & 46.9 & 8.3 & 34.6 & 12.6 \\
\hline & & 29.5 & 1.30 & 4.65 & 35.5 & 23.4 & 214 & 66.5 & 9.5 & 56.9 & 17.4 \\
\hline & SD & \pm 1.89 & \pm 0.09 & \pm 0.28 & \pm 5.2 & \pm 4.18 & \pm 52 & \pm 9.6 & \pm 0.92 & \pm 11.2 & \pm 2.33 \\
\hline
\end{tabular}

* Column headings are as in Table II.

$\dagger$ Figures above and below the mean value indicate the range of observations.

This relationship in Subjects DVB, CJV and RED is shown in Figure 1. The slope of the lines reflects the $V_{c}$ component, and from the intercept, DM is calculated. The general linearity of the points in any one individual is clear from this figure. The values of $V_{c}$ and $D_{m}$ calculated from these slopes appear in Table II. The data in Figure 1 have been used to predict the DL at different values of $\overline{\mathrm{P}} \mathrm{C}_{\mathrm{O}_{2}}$ in the same three normal subjects during hypoxia, during voluntary hyper- ventilation, and during $\mathrm{CO}_{2}$ breathing. In Table $\mathrm{V}$ are shown the results of measurements of DL under these conditions in which little deviation could be demonstrated from the values of DL predicted on the basis of Figure 1. This conclusion is discussed in detail later.

Patients. In Table VI are shown the physical characteristics of the first two groups of patients studied. Group 1 consisted of three patients who had had various parts of the lung removed, with

TABLE V

Effect of hyperventilation and hypoxia during exercise at $3 \mathrm{mph}$ on a flat surface

\begin{tabular}{|c|c|c|c|c|c|c|c|}
\hline Subject & $\begin{array}{c}\text { Rate and } \\
\text { grade }\end{array}$ & Experiment & Ventilation & $\begin{array}{c}\text { Inspired } \\
\text { oxygen\% } \\
\text { FIO2 }\end{array}$ & $\begin{array}{l}\text { Mean } \\
\text { capillary } \\
\mathrm{O}_{2} \\
\overline{\mathrm{P}}_{\mathrm{CO} 2}\end{array}$ & $\begin{array}{l}\text { Measured } \\
\text { DLCO }\end{array}$ & $\begin{array}{l}\text { Predicted } \\
\text { DLCo* }\end{array}$ \\
\hline \multirow{3}{*}{ DVB } & \multirow{3}{*}{$\begin{array}{l}3 \mathrm{mph} \\
\text { Flat }\end{array}$} & & $L / \min$ & & & $\begin{array}{c}m l \mathrm{CO} / \\
\min / \\
m m \mathrm{Hg}\end{array}$ & \\
\hline & & Hypoxia & 44.3 & 10.7 & 22.0 & 41.8 & 41.2 \\
\hline & & $\begin{array}{l}\text { Hypoxia } \\
\text { Hypoxia } \\
\text { Hypoxia } \\
\text { Hyperventilation } \\
5.8 \% \mathrm{CO}_{2}\end{array}$ & $\begin{array}{l}40.5 \\
35.6 \\
32.3 \\
47.5 \dagger \\
48.3 \dagger\end{array}$ & $\begin{array}{l}12.4 \\
12.8 \\
15.0 \\
20.8 \\
20.8\end{array}$ & $\begin{array}{r}30.9 \\
34.9 \\
39.9 \\
96.3 \\
106.7\end{array}$ & $\begin{array}{l}43.5 \\
39.4 \\
32.6 \\
36.0 \\
34.5\end{array}$ & $\begin{array}{l}40.6 \\
39.7 \\
39.3 \\
34.8 \\
33.9\end{array}$ \\
\hline \multirow[t]{2}{*}{ RED } & \multirow{2}{*}{$\begin{array}{l}3 \mathrm{mph} \\
\text { Flat }\end{array}$} & Hypoxia & 37.4 & 11.0 & 29.5 & 52.2 & 42.5 \\
\hline & & $\begin{array}{l}\text { Hypoxia } \\
\text { Hyperventilation } \\
5.8 \% \mathrm{CO}_{2}\end{array}$ & $\begin{array}{l}39.6 \\
51.3 \dagger \\
56.1 \dagger\end{array}$ & $\begin{array}{l}11.6 \\
20.8 \\
20.8\end{array}$ & $\begin{array}{r}30.2 \\
102.8 \\
95.5\end{array}$ & $\begin{array}{l}47.4 \\
43.8 \\
46.4\end{array}$ & $\begin{array}{l}42.3 \\
36.7 \\
37.7\end{array}$ \\
\hline \multirow[t]{2}{*}{ CJV } & \multirow{2}{*}{$\begin{array}{l}3 \mathrm{mph} \\
\text { Flat }\end{array}$} & Hypoxia & 33.7 & 10.8 & 25.0 & 40.4 & 44.8 \\
\hline & & $5.8 \% \mathrm{CO}_{2}$ & $61.5 \dagger$ & 20.8 & 113.2 & 45.0 & 40.7 \\
\hline
\end{tabular}

* This column indicates the DLco predicted for the individual at the measured $\bar{P}_{02}$ (see Figure 1 ).

† See Table II for results at normal ventilations in these subjects. 
TABLE VI

Physical characteristics and resting pulmonary function tests in Groups 1 and 2 of the patients studied*

\begin{tabular}{|c|c|c|c|c|c|c|c|c|c|c|}
\hline No. & Subject & Sex & Age & $\mathrm{Ht}$ & $\mathrm{Wt}$ & vc & FRC & ME\% & MMFR & Diagnosis \\
\hline \multirow{2}{*}{\multicolumn{11}{|c|}{ Group 1}} \\
\hline & & & & & & & & & & \\
\hline $\begin{array}{l}15 \\
16 \\
17\end{array}$ & $\begin{array}{l}\text { M FEN } \\
\text { J COT } \\
\text { V RIC }\end{array}$ & $\begin{array}{l}\mathrm{F} \\
\mathrm{M} \\
\mathrm{F}\end{array}$ & $\begin{array}{l}50 \\
41 \\
27\end{array}$ & $\begin{array}{l}65 \frac{1}{2} \\
69 \frac{1}{2} \\
68 \frac{1}{2}\end{array}$ & $\begin{array}{l}133 \\
157 \frac{1}{2} \\
96 \frac{1}{2}\end{array}$ & $\begin{array}{l}1.98 \\
2.12 \\
1.41\end{array}$ & $\begin{array}{l}1.27 \\
1.87 \\
2.13\end{array}$ & $\begin{array}{l}39 \\
47 \\
33\end{array}$ & $\begin{array}{l}1.80 \\
1.50 \\
0.52\end{array}$ & $\begin{array}{l}\text { Pneumonect. L } \\
\text { Pneumonect. R } \\
\text { Use of only rt. upper lobe }\end{array}$ \\
\hline \multicolumn{11}{|c|}{ Group 2} \\
\hline $\begin{array}{l}18 \\
19 \\
20 \\
21 \\
22 \\
23 \\
24 \\
25\end{array}$ & $\begin{array}{l}\text { A MAD } \\
\text { G KOS } \\
\text { F BOU } \\
\text { I GAL } \\
\text { F ATK } \\
\text { T NAD } \\
\text { A McC } \\
\text { H WAH }\end{array}$ & $\begin{array}{l}F \\
F \\
M \\
F \\
F \\
M \\
M \\
M\end{array}$ & $\begin{array}{l}38 \\
32 \\
35 \\
22 \\
30 \\
20 \\
37 \\
25\end{array}$ & $\begin{array}{l}65 \\
65 \frac{1}{2} \\
64 \frac{1}{4} \\
63 \\
63 \frac{1}{4} \\
69 \\
71 \\
71 \frac{1}{2}\end{array}$ & $\begin{array}{l}122 \\
120 \\
132 \\
78 \\
100 \frac{1}{2} \\
155 \\
152 \\
149\end{array}$ & $\begin{array}{l}2.80 \\
2.20 \\
2.35\end{array}$ & $\begin{array}{l}2.72 \\
2.42 \\
2.42\end{array}$ & $\begin{array}{l}64 \\
60 \\
64\end{array}$ & $\begin{array}{l}1.31 \\
2.70 \\
2.25\end{array}$ & $\begin{array}{l}\text { Thyrotoxicosis } \\
\text { Thyrotoxicosis } \\
\text { Atr. sept. defect } \\
\text { Atr. sept. defect } \\
\text { Pat. duct. art. } \\
\text { Pat. düct. art. } \\
\text { Pat. duct. art. } \\
\text { Tric. atresia }\end{array}$ \\
\hline
\end{tabular}

* See Table I for details of column headings.

a reduction in FRC as a consequence. Group 2 consisted of a group of eight patients in all of whom there was reason to suppose that the pulmonary blood flow was abnormal; two of these patients had untreated thyrotoxicosis, two had atrial septal defects with high pulmonary blood flows, three had a patent ductus arteriosus with considerable increase in pulmonary flow in each case, and the last patient had tricuspid atresia with a reduction in cardiac output at rest as shown by cardiac catheterization. The results of determinations of $V_{c}$ and $D_{M}$ on these patients are shown in Table VII. In each case the exercise managed by the patient is shown in the second column and the minute volume and oxygen uptake are adjacent. The two patients who have had a pneumonectomy showed considerable variation in pulmonary capillary blood volume, though both values may be considered to be within half the approximate normal range. The membrane components are very constant and again are about half the predicted normal values for these subjects. The membrane component in relation to ventilated lung volume is slightly below the predicted values in these patients and the $V_{c}$ in relation to lung volume appears to be normal in the first two patients and definitely reduced in the third (Patient 17). These findings are of some interest since

TABLE VII

Results of determinations of pulmonary capillary blood volume and membrane diffusion components in patients in Groups 1 and 2*

\begin{tabular}{|c|c|c|c|c|c|c|c|c|c|c|c|c|}
\hline Subject & Diagnosis & Rate† & $\underset{\text { vol }}{\text { Minute }}$ & $\dot{\mathrm{V}}_{2}$ & $\begin{array}{l}\dot{V}_{\mathbf{V}_{2}} / \\
\mathbf{V}_{\text {air }}\end{array}$ & $\begin{array}{l}\text { DLco } \\
\text { (air) }\end{array}$ & $\begin{array}{l}\text { DLCo } \\
\left(\mathrm{O}_{2}\right)\end{array}$ & $\mathrm{V}_{\mathrm{c}}$ & $\mathrm{DM}_{\mathrm{M}}$ & DLCo/ & $\begin{array}{l}\mathrm{V}_{\mathrm{c}} / \mathrm{f} \\
\mathrm{MC}\end{array}$ & $\begin{array}{l}\text { DM/ } \\
\text { MC }\end{array}$ \\
\hline Group 1 & & $m p h$ & $L / \min$ & $L / \min$ & & & & $m l$ & & & & \\
\hline \multirow{2}{*}{$\begin{array}{l}15 \mathrm{M} \text { FEN } \\
16 \mathrm{~J} \text { COT } \\
17 \mathrm{VIC}\end{array}$} & \multirow{2}{*}{$\begin{array}{l}\text { Pneumonect. L. } \\
\text { Pneumonect. R. } \\
\text { Use of only rt. } \\
\text { upper lobe }\end{array}$} & 2.5 & $\begin{array}{l}19.90 \\
21.60\end{array}$ & $\begin{array}{l}0.80 \\
1.05\end{array}$ & $\begin{array}{l}4.00 \\
4.85\end{array}$ & $\begin{array}{l}12.3 \\
16.7\end{array}$ & $\begin{array}{r}6.5 \\
11.1\end{array}$ & $\begin{array}{r}47 \\
103\end{array}$ & $\begin{array}{l}14.9 \\
19.3\end{array}$ & $\begin{array}{l}6.3 \\
7.3\end{array}$ & $\begin{array}{l}24.0 \\
44.0\end{array}$ & $\begin{array}{l}7.6 \\
8.4\end{array}$ \\
\hline & & 1.5 & 15.67 & 0.53 & 3.38 & 11.5 & 6.0 & 38 & 18.3 & 4.7 & 15.3 & 7.5 \\
\hline \multicolumn{2}{|c|}{ Approx normal } & 3 & $21-31$ & $1.0-1.9$ & $3.5-4.8$ & $22-45$ & $12-27$ & $68-200$ & $33-66$ & $6.9-10.5$ & $24-56$ & $9-17$ \\
\hline \multicolumn{13}{|l|}{ Group 2} \\
\hline $\begin{array}{l}18 \text { A MAD } \\
19 \text { G KOS } \\
20 \text { F BOU } \\
21 \text { I GAL } \\
22 \text { F ATK } \\
23 \text { T NAD } \\
24 \text { A McC } \\
25 \text { H WAH }\end{array}$ & $\begin{array}{l}\text { Thyrotoxicosis } \\
\text { Thyrotoxicosis } \\
\text { Atr. sept. defect } \\
\text { Atr. sept. defect } \\
\text { Pat. duct. art. } \\
\text { Pat. duct. art. } \\
\text { Pat. duct. art. } \\
\text { Tric. atresia }\end{array}$ & $\begin{array}{l}1.5 \\
2 \\
1.5 \\
2.5 \\
1.5 \\
2 \\
1.75 \\
2\end{array}$ & $\begin{array}{l}18.65 \\
20.50 \\
21.60 \\
22.65 \\
17.30 \\
24.85 \\
35.60 \\
34.10\end{array}$ & $\begin{array}{l}0.73 \\
0.88 \\
0.60 \\
0.55 \\
0.54 \\
0.97 \\
1.09 \\
0.62\end{array}$ & $\begin{array}{l}3.91 \\
4.29 \\
2.78 \\
2.43 \\
3.12 \\
3.90 \\
3.06 \\
1.82\end{array}$ & $\begin{array}{l}17.3 \\
19.5 \\
31.0 \\
30.2 \\
21.5 \\
22.7 \\
21.9 \\
23.9\end{array}$ & $\begin{array}{l}11.6 \\
11.0 \\
19.6 \\
18.6 \\
13.2 \\
14.1 \\
13.7 \\
12.7\end{array}$ & $\begin{array}{r}111 \\
79 \\
164 \\
150 \\
112 \\
113 \\
109 \\
83\end{array}$ & $\begin{array}{l}21.2 \\
27.6 \\
41.7 \\
41.7 \\
27.6 \\
30.0 \\
29.0 \\
40.0\end{array}$ & $\begin{array}{r}6.9 \\
7.1 \\
9.9 \\
10.4 \\
7.6 \\
6.8 \\
5.5 \\
7.0\end{array}$ & $\begin{array}{l}44.3 \\
28.9 \\
52.4 \\
51.5 \\
39.7 \\
34.0 \\
36.3 \\
24.3\end{array}$ & $\begin{array}{r}8.5 \\
10.1 \\
13.3 \\
14.4 \\
9.8 \\
9.0 \\
7.5 \\
11.7\end{array}$ \\
\hline
\end{tabular}

* See previous tables for details of column headings.

$\dagger$ All estimates done on exercise at rate shown on a flat surface. 
TABLE VIII

Physical data and resting pulmonary function tests in patients in Groups 3 and 4*

\begin{tabular}{lllllllllll}
\hline \hline No. & Subject & Sex & Age & Ht & Wt & VC & FRC & ME\% & MMFR & Diagnosis \\
\hline & Group 3 & & & in. & $l b s$ & $L$ & $L$ & & L/sec & \\
26 & M LED & F & 31 & 63 & 150 & & & & & \\
27 & H LAP & F & 42 & $59 \frac{1}{2}$ & 79 & 1.95 & 2.34 & 40 & 1.27 & MS \\
28 & E MOF & F & 34 & $57 \frac{3}{4}$ & 102 & 2.50 & 2.37 & 67 & 3.50 & MS \\
29 & M HEA & F & 46 & 62 & 104 & 2.16 & 2.51 & 59 & 2.20 & MS I I \\
30 & P MOL & M & 19 & 70 & 155 & 3.94 & 2.83 & 50 & 5.70 & MS I \\
& Group 4 & & & & & & & & & \\
31 & E RYL & F & 50 & 61 & $117 \frac{1}{2}$ & 1.45 & 1.58 & 48 & 1.40 & DIF \\
32 & E St.L & M & 50 & 65 & 170 & 3.42 & 3.26 & 43 & 1.20 & DIF \\
33 & V AYB & F & 48 & 64 & 140 & 1.87 & 1.41 & 59 & 2.40 & DIF \\
34 & S ARN & F & 64 & 65 & 162 & 1.43 & 1.75 & 59 & 0.75 & DIF \\
35 & T LAV & F & 46 & 63 & 113 & 1.70 & 2.38 & 52 & 2.50 & BS \\
36 & D BOU & F & 15 & 67 & 116 & 2.75 & 3.07 & 63 & 2.70 & BS \\
\hline
\end{tabular}

* See Table I for details of column headings. $M S=$ mitral stenosis; $M S+I=$ mitral stenosis + insufficiency; $\mathrm{DIF}=$ diffuse interstitial fibrosis $; \mathrm{BS}=$ Boeck sarcoid.

there is no evidence that the $\mathrm{V}_{\mathrm{c}}$ of the remaining lung approximates that of the two lungs, although presumably it has a high pulmonary blood flow. The membrane components have been universally lowered by removal of one lung, as would be expected. Patients 18 to 24 may be presumed to have an increased pulmonary flow. The cardiac output is known to be much increased in untreated thyrotoxicosis during exercise (12), and a high resting pulmonary flow had been confirmed by cardiac catheterization in Patients 20 to 24 . $\mathrm{Pa}$ tient 25 with tricuspid atresia was found at cardiac catheterization to have a cardiac index of half the predicted value. Consideration of the results in
Group 2 as a whole shows that there is no evidence that an abnormally high or low pulmonary blood flow during exercise causes a change in $V_{c}$ since all measurements of $V_{c}$ in this group are within the predicted range. It is perhaps of interest that the membrane diffusing components (DM/MC) were somewhat lower than expected in Patients 18 and 24, which might suggest that in some of these conditions there is an impairment of diffusion across the membrane resulting from the persistent high blood flow. In Table VIII are shown the physical characteristics and results of resting pulmonary function tests in patients in Group 3 and 4. Group 3 consists of four pa-

TABLE IX

Results of determinations of pulmonary capillary blood volume and membrane diffusion component in patients in Groups 3 and $4^{*}$

\begin{tabular}{|c|c|c|c|c|c|c|c|c|c|c|c|c|}
\hline Subject & Diagnosis & Rate† & $\underset{\text { vol }}{\text { Minute }}$ & $\dot{\mathrm{V}}_{2}$ & $\begin{array}{l}\dot{\mathrm{V}}_{\mathrm{O}_{2}}^{\prime} \\
\dot{\mathrm{V}}_{\mathrm{air}}\end{array}$ & $\begin{array}{l}\text { DLco } \\
\text { (air) }\end{array}$ & $\begin{array}{l}\mathrm{DV}_{\mathrm{CO}} \\
\left(\mathrm{O}_{2}\right)\end{array}$ & $V_{c}$ & DM & $\begin{array}{l}\text { DLCo/ } \\
\text { MC }\end{array}$ & $\begin{array}{l}\mathrm{V}_{c /} \\
\mathrm{MC}\end{array}$ & $\begin{array}{l}\mathrm{DM} / \\
\mathrm{MC}\end{array}$ \\
\hline \multicolumn{13}{|l|}{ Group 3} \\
\hline $\begin{array}{l}26 \text { M LED } \\
27 \text { H LAP } \\
28 \text { E MOF } \\
28 \text { E MOF } \\
29 \text { M HEA } \\
30 \text { P MOL }\end{array}$ & $\begin{array}{l}\text { MS } \\
\text { MS } \\
\text { MS } \\
\text { MS post op. } \\
\text { MS } \\
\text { MS, MI }\end{array}$ & $\begin{array}{l}1.5 \\
2 \\
1.5 \\
1.5 \\
1 \\
2\end{array}$ & $\begin{array}{l}19.80 \\
18.80 \\
20.16 \\
13.63 \\
26.60 \\
22.35\end{array}$ & $\begin{array}{l}0.82 \\
0.53 \\
0.64 \\
0.60 \\
0.53 \\
0.94\end{array}$ & $\begin{array}{l}4.12 \\
2.82 \\
3.17 \\
4.36 \\
2.00 \\
4.19\end{array}$ & $\begin{array}{l}26.9 \\
12.9 \\
18.7 \\
21.3 \\
14.3 \\
25.6\end{array}$ & $\begin{array}{r}17.4 \\
11.3 \\
15.4 \\
11.9 \\
7.7 \\
13.8\end{array}$ & $\begin{array}{r}152 \\
293 \\
275 \\
84 \\
50 \\
93\end{array}$ & $\begin{array}{l}34.8 \\
13.7 \\
20.5 \\
31.3 \\
23.0 \\
38.7\end{array}$ & $\begin{array}{r}10.0 \\
4.8 \\
6.9 \\
8.1 \\
4.8 \\
7.7\end{array}$ & $\begin{array}{c}56.4 \\
110.0 \\
101.5 \\
31.1 \\
16.9 \\
28.3\end{array}$ & $\begin{array}{r}12.9 \\
5.1 \\
7.6 \\
11.5 \\
7.7 \\
11.8\end{array}$ \\
\hline \multicolumn{2}{|c|}{ Approx normal } & 3 & $21-31$ & $1.0-1.9$ & $3.5-4.8$ & $22-45$ & $12-27$ & $68-200$ & $33-66$ & $6.9-10.5$ & $24-56$ & $9-17$ \\
\hline \multicolumn{13}{|l|}{ Group 4} \\
\hline $\begin{array}{l}31 \text { E RYL } \\
32 \text { E St.L } \\
33 \text { V AYB } \\
35 \text { S ARN } \\
35 \text { T LAV } \\
36 \text { D BOU }\end{array}$ & $\begin{array}{l}\text { CDIF } \\
\text { CDIF } \\
\text { CDIF } \\
\text { CDIF } \\
\text { BS } \\
\text { BS }\end{array}$ & $\begin{array}{l}1.5 \\
1.75 \\
2 \\
0.75 \\
1.5 \\
2\end{array}$ & $\begin{array}{l}15.20 \\
27.80 \\
30.00 \\
24.50 \\
20.40 \\
16.55\end{array}$ & $\begin{array}{l}0.61 \\
1.13 \\
0.69 \\
0.59 \\
0.72 \\
0.77\end{array}$ & $\begin{array}{l}4.01 \\
4.07 \\
2.30 \\
2.41 \\
3.53 \\
4.66\end{array}$ & $\begin{array}{r}8.9 \\
15.2 \\
7.9 \\
6.8 \\
17.8 \\
21.1\end{array}$ & $\begin{array}{r}7.8 \\
11.4 \\
4.8 \\
3.5 \\
6.8 \\
12.0\end{array}$ & $\begin{array}{r}21 \\
140 \\
36 \\
20 \\
36 \\
84\end{array}$ & \begin{tabular}{r|}
9.4 \\
17.2 \\
10.4 \\
10.4 \\
45.9 \\
30.0
\end{tabular} & $\begin{array}{l}6.0 \\
3.8 \\
4.4 \\
3.3 \\
6.4 \\
8.6\end{array}$ & \begin{tabular}{r|}
13.7 \\
35.0 \\
19.6 \\
9.8 \\
13.0 \\
34.3
\end{tabular} & $\begin{array}{r}6.3 \\
4.3 \\
5.7 \\
5.0 \\
16.5 \\
12.3\end{array}$ \\
\hline
\end{tabular}

* See previous tables for details of column headings. MS = mitral stenosis; CDIF $=$ chronic diffuse interstitial fibrosis; BS $=$ Boeck sarcoid. $\dagger$ All estimates done on exercise at rate shown on a flat surface, except those for Subject 36 who exercised on a surface with a 5 per cent slope. 
tients with mitral stenosis and one with mitral stenosis with predominant mitral insufficiency. In Group 4 are four patients with chronic diffuse interstitial pulmonary fibrosis (in whom the diagnosis has been confirmed by lung biopsy in three), and two patients with sarcoid of the lung (in both of whom the diagnosis has been confirmed by lung biopsy). Table IX shows the results of determination of $V_{c}$ and membrane components in these patients. In Patients 27 and 28, both of whom represented typical examples of tight mitral stenosis with considerable dyspnea and a reduced pulmonary compliance, the values of $\mathrm{V}_{\mathrm{c}}$ are high, and those for the DM are low. Patient 28 was studied three weeks after a successful valvotomy, at which a very tight valve was found. On each occasion the level of exercise was the same, and there was an increase in $\mathrm{Vo}_{2} / \mathrm{V}_{\text {air }}$ after surgery from 3.17 to 4.36 , in conformity with the observations of Cotes (13), and MacIntosh, Sinnott, Milne and Reid (14). There was a slight increase in over-all DL from 18.7 to 21.3 , but this small change masks considerable alterations in $\mathrm{V}_{\mathrm{c}}$ and DM. The $\mathrm{V}_{\mathrm{c}}$ fell from 275 to $85 \mathrm{ml}$ after surgery, and the membrane component rose from 20.5 to 31.3 . Patient 29 had the murmur of mitral stenosis, but was judged to be so little incapacitated that surgery was not indicated. The pulmonary compliance in this patient was within normal limits $(0.190 \mathrm{~L}$ per $\mathrm{cm} \mathrm{H}_{2} \mathrm{O}$ ). It is of particular interest, therefore, that the $V_{c}$ was normal, although there was a slight reduction in DM. In Patient 30, who had predominant mitral incompetence, the values for $\mathrm{V}_{\mathrm{c}}$ and $\mathrm{DM}_{\mathrm{M}}$ were within normal limits.

In Group 4 the changes are those one would expect to find in patients whose main difficulty is the transfer of oxygen across the pulmonary membrane. The $D_{M}$ is reduced in all patients in the pure pulmonary fibrosis group (Patients 31 to 34 ), and the extent of this reduction can be seen from comparison of DM in relation to ventilated lung volume in the last column. The values found in these four patients vary between 4.3 and 6.3 for this figure $(\mathrm{Dm} / \mathrm{MC})$ whereas the normal range is from 9 to 17 , approximately. Patients 35 and 36 , with sarcoidosis, show considerable variation, the former having a normal $\mathrm{DM}_{\mathrm{M}}$ but a low value of $\mathrm{V}_{c}$, and the latter having a normal $\mathrm{V}_{c}$ and $\mathrm{a}$ nearly normal DM. These variations in this condition are to be expected, and the low $V_{c}$ found in
Patient 35 may reflect the fact that the main brunt of the lesion has been perivascular, as has been suggested by a number of authors $(15,16)$ for this condition. It is of interest, however, that the considerable difference in $V_{c}$ between these two patients is not reflected by much difference in the over-all DL.

\section{DISCUSSION}

The data so far reported on the subdivision of the pulmonary diffusing capacity into its two components have been obtained by use of a single breath method (5-7). Apart from the objection that the holding of a deep breath may modify the quantities it is desired to measure, the single breath is difficult to manage during exercise. It is now generally recognized that the mean alveolar $\mathrm{CO}$ tension may be reliably measured during exercise in a steady state experiment, at least in subjects with normal gas distribution (8).

The present studies show that the components of pulmonary diffusion capacity may be reliably measured by this technique during steady state exercise, and the theoretical work of Forster and his colleagues has been confirmed by the demonstration under these conditions of a linear relationship between $1 / \mathrm{DL}$ and $1 / \theta$ (see Figure 1 ). It seems likely that the differences in the rate of gas diffusion between individuals at the same rate of exercise are mainly attributable to variations in the $\mathrm{V}_{\mathrm{c}}$. The DM, when expressed in relation to the volume of lung being ventilated, appears to be relatively constant in normal subjects at the same exercise level. The relative importance of these two factors is well illustrated by comparison of normal Subjects 2 and 6 in Table III. At the same exercise rate, the over-all DL was 12 units greater in Subject CJV; $V_{c}$ in CJV was approximately twice that in Subject 6, yet the DM expressed in relation to lung volume was only 1 unit different between these two subjects. There was a large difference in $V_{c}$ expressed in relation to the ventilated lung volume illustrating that the principal difference between these two subjects lay in this component rather than in the membrane component. Although these two subjects were the same age (see Table I), there was a 6 inch difference in height and a 60 pound difference in weight between them. There was only a $600 \mathrm{ml}$ difference in vital capacity and a $700 \mathrm{ml}$ difference in 
FRC. The constancy of the results obtained in duplicate estimates of $\mathrm{V}_{\mathrm{c}}$ and membrane components in the present study (Table II) may be compared to that reported by Lewis and associates (6) using the single breath technique. His data show less variation in $V_{c}$ on repeat determinations, but the Dм showed more variation than that found in the present study. The reason for these differences is not altogether clear, but may reside in the errors inherent in the two techniques.

It may be pointed out, however, that provided the tidal volume is comparable during the two determinations of $D_{L}$, the value assumed for the respiratory dead space exerts no effect on the calculated $\mathrm{V}_{\mathrm{c}}$. Consequently, with a steady state technique of the type used, this subdivision of the DL may be more reliably measured than the calculated DM, which is affected by the value assumed for the dead space.

Ross, Frayser and Hickam (17), using the Filley method of calculating the mean alveolar $\mathrm{CO}$ tension, reported large increases in $\mathrm{DL}_{\mathrm{co}}$ with hyperventilation. These authors also suggested that much of the increase in $\mathrm{DL}_{\mathrm{CO}}$ on exercise is to be attributed directly to the ventilation change. The data in Table $\mathrm{V}$ indicate that the observed values of $\mathrm{DL}_{\mathrm{co}}$ under conditions of hypoxia, voluntary hyperventilation, or $\mathrm{CO}_{2}$ breathing, differ little from those that would be predicted for the individual concerned at the particular alveolar oxygen tension obtaining during the experiments. In only one subject (RED) did the observed values fall consistently above the predicted figures. No effect was demonstrable in the other two subjects. These results suggest that during exercise, neither hypoxia nor an increase in ventilation per se, causes any consistent or considerable alteration in the state of the pulmonary bed. The discrepancy between these observations and those of Ross and co-workers (17) and Turino, Brandfonbrener and Fishman (18), are not readily explained. Both authors reported striking increases in DL during hyperventilation when the alveolar $\mathrm{CO}$ tension was computed from the measured arterial $\mathrm{CO}_{2}$ tension. Such increases are not found if the end tidal $\mathrm{CO}$ is directly measured or if the mean alveolar $\mathrm{CO}$ is calculated, using a predicted value of respiratory dead space.

Forster (5) considered that anoxia might well change the pulmonary capillary bed, but if this were the case, the observed $\mathrm{DL}_{\mathrm{Co}}$ in Table $\mathrm{V}$ under these conditions might be measurably greater than that predicted on the basis of the linear relationship shown in Figure 1. The precision of the data in Figure 1 in respect to these three normal subjects suggests that the line of observed relationship between $1 / \mathrm{DL}$ and $1 / \theta$ can be extrapolated confidently to cover low oxygen tensions, though $\theta$ values may not be reliable at capillary oxygen tensions below $150 \mathrm{~mm} \mathrm{Hg} \mathrm{(2).} \mathrm{The} \mathrm{finding} \mathrm{of}$ little discrepancy between observed and predicted figures indicates that the effect of anoxia is probably slight, at least in short-term experiments on exercise, but more precise conclusions cannot be drawn from the data at this stage.

The study of the 22 patients in the present series leads to the following general conclusions.

1. When portions of the lungs are removed, $D_{M}$ is proportionately reduced. The $V_{c}$ of the remaining lung seems variable. The general result is that the over-all DL is about that predicted for one lung, as was found in previous studies of Mcllroy and Bates (19) and Linderholm (20). There does not appear to be any considerable increase in the $\mathrm{V}_{\mathrm{c}}$ of the remaining lung in relation to its gas volume, although presumably the pulmonary blood flow through the lung is substantially increased.

2. In long-standing conditions in which the pulmonary blood flow is greatly increased, there is no concomitant increase in $\mathrm{V}_{\mathrm{c}}$. This conclusion is somewhat at variance with that of Auchincloss, Gilbert and Eich (21), who used the single breath De technique at rest to study patients with congenital and acquired heart disease. These authors found an increased DL at rest in some patients in the congenital heart disease group, but they did not report any exercise results. Their findings are therefore not necessarily at variance with the present conclusion that the exercise $\mathrm{DM}_{\mathrm{M}}$ and $\mathrm{V}_{\mathrm{c}}$ are not increased in these patients compared with those of normal subjects. The possibility exists that the taking and holding of a single breath by patients with large intracardiac shunts may significantly alter the state of the pulmonary bed. The finding of a lowered DM in Patients 23 and 24 may well reflect irreversible changes secondary to the pulmonary hypertension, since the two patients with primary pulmonary hypertension studied by McNeill and associates (7) showed a de- 
crease both in DM and in $\mathrm{V}_{\mathrm{c}}$. The pulmonary diffusing capacity in thyrotoxicosis has been noted previously to be normal or lower than normal, even though the pulmonary blood flow is considerably elevated. ${ }^{1}$ The two patients with untreated thyrotoxicosis included in the present study illustrate this finding. The predicted $\mathrm{DL}_{\mathrm{CO}}$ for these two patients at the exercise level they maintained is 27 and $31 \mathrm{ml} \mathrm{CO}$ per minute per $\mathrm{mm} \mathrm{Hg}$, respectively (8), whereas the measured figures were 17.3 and 19.5. The DM was reduced in the first of these patients and was clearly limiting the rate of gas diffusion considerably. In the second patient it was just below the approximate normal level. In neither patient was there any increase in $V_{c}$. Taken together, the findings in Group 2 in Table VII indicate that the components of pulmonary diffusion are independent of the absolute level of pulmonary blood flow. This supports the animal experimentation reported by Rosenberg and Forster in which the authors arrived at the same conclusion (22).

3. The finding of high values for $V_{c}$ in mitral stenosis supports the conclusion of $\mathrm{McNeill}$ and associates (7). The additional evidence presented above suggests that this may be an important measurement in this condition, since the over-all DL may alter little after a successful valvotomy and so conceal the fact that the $V_{c}$ and $D_{M}$ components have changed considerably in different directions. The variable results noted by others $(14,23,24)$ in the over-all DL in mitral stenosis, may well be explained by the present observation that further subdivision of the DL is required before the data become meaningful in terms of changes in the pulmonary capillary bed. Further, the present finding of normal values in a patient with little disability, who was known to have a normal pulmonary compliance, certainly suggests that alterations in $V_{c}$ and membrane diffusion components represent a basic change in the physiopathology of this disease. The demonstration of a marked change in these values only three weeks after a valvotomy (Patient 28, Table IX), is also of considerable interest, since it has proved difficult to correlate the considerable subjective improvement which many such patients have with any measurable physiological variable.

\footnotetext{
1 Bates, D. V. Unpublished observations.
}

It may be that the measured $V_{c}$ during exercise will prove to be a sensitive indicator of change occurring in the pulmonary bed as a result of valvotomy.

4. In pulmonary fibrosis the measurement of DM probably represents the most sensitive criterion of normality available. Its expression in terms of the ventilated lung (DM/MC) increases its sensitivity considerably. This is well illustrated by Patient 32 in Table IX whose pulmonary infiltration was known to have been present for less than three months. The DM/MC ratio was 4.3 against the predicted value of at least 9.0. The lung biopsy in this patient showed that the alveolar walls were uniformly thickened but only by about one extra layer of cells. Pathologically the section was regarded as representing an extremely early example of Hamman-Rich syndrome. Systematic study of $\mathrm{DM}_{\mathrm{M}}$ changes in this and other types of pulmonary fibrosis would undoubtedly provide a sensitive indicator of alterations in the pulmonary membrane. In certain diseases such as sarcoidosis, it may be found that the main area of involvement in the lungs can be localized by a study of the relative changes in $V_{c}$ and DM. The finding in the present study of an unexpectedly low $\mathrm{V}_{\mathrm{c}}$ in a patient with sarcoidosis, as well as in two of the patients with a diffuse interstitial fibrosis, suggests that the involvement of the lung may be quite different in different patterns of these diseases.

\section{SUM MARY}

1. The components of the pulmonary diffusing capacity have been studied in 14 normal individuals, and in a group of 22 patients with varying clinical conditions. All the determinations of pulmonary capillary blood volume and membrane diffusing capacity were made during exercise.

2. The results reported show that this determination is sufficiently stable and reliable to permit comparisons to be made between normal subjects and patients.

3. The data suggest that in normal subjects the pulmonary diffusing capacity increases with exercise mainly because the pulmonary capillary blood volume rises. Individual differences in diffusing capacity also seem mainly attributable to variations in this component. Hyperventilation and hypoxia 
could not be shown to exert much influence on the pulmonary diffusing capacity.

4. Removal of one lung appears to result in a halving of the membrane component.

5. High flow conditions in the lesser circulation are not associated with any increase in pulmonary capillary blood volume. In one patient with a reduced pulmonary flow the pulmonary capillary blood volume was normal.

6. Mitral stenosis may lead to a considerable increase in pulmonary capillary blood volume, and a concomitant reduction in membrane component. In one patient studied before and after a successful valvotomy, the pulmonary capillary blood volume at equivalent exercise had dropped from 275 $\mathrm{ml}$ before operation to $85 \mathrm{ml}$ postoperatively and the membrane component rose from 20 to $31 \mathrm{ml}$ $\mathrm{CO}$ per minute per $\mathrm{mm} \mathrm{Hg}$. This reciprocal change in these two variables resulted in a very similar over-all diffusing capacity before and after operation.

7. In a group of patients with pulmonary fibrosis the main defect was found to be in the membrane diffusion component, the pulmonary capillary blood volume being either normal or reduced.

8. It is concluded that this technique based on the theoretical work of Forster (5) is capable of yielding valuable information in a wide variety of clinical conditions.

\section{ACKNOWLEDGMENTS}

We wish to thank members of the Medical and Surgical Staff of the Royal Victoria Hospital who referred patients to us for study. Mr. L. S. Bartlett, Mr. L. D. Pengelly and Mr. L. Nowaczek provided valuable technical assistance during this work.

\section{REFERENCES}

1. Forster, R. E., Roughton, F. J. W., Cander, L., Briscoe, W. A., and Kreuzer, F. Apparent pulmonary diffusing capacity for $\mathrm{CO}$ at varying alveolar $\mathrm{O}_{2}$ tensions. J. appl. Physiol. 1957, 11, 277.

2. Roughton, F. J. W., and Forster, R. E. Relative importance of diffusion and chemical reaction rates in determining the rate of exchange of gases in the human lung. J. appl. Physiol. 1957, 11, 290.

3. Forster, R. E., Roughton, F. J. W., Kreuzer, F., and Briscoe, W. A. Photocolorimetric determination of the rate of uptake of $\mathrm{CO}$ and $\mathrm{O}_{2}$ by reduced human red cell suspensions at $37^{\circ} \mathrm{C}$. J. appl. Physiol. 1957, 11, 260.
4. Roughton, F. J. W., Forster, R. E., and Cander, L. Rate at which $\mathrm{CO}$ replaces $\mathrm{O}_{2}$ from combination with human hemoglobin in solution and in the red cell. J. appl. Physiol. 1957, 11, 269.

5. Forster, R. E. Exchange of gases between alveolar air and pulmonary capillary blood: Pulmonary diffusing capacity. Physiol. Rev. 1957, 37, 391.

6. Lewis, B. M., Lin, T. H., Noe, F. E., and Komisaruk, $R$. The measurement of pulmonary capillary blood volume and pulmonary membrane diffusing capacity in normal subjects; the effects of exercise and position. J. clin. Invest. 1958, 37, 1061.

7. McNeill, R. S., Rankin, J., and Forster, R. E. The diffusing capacity of the pulmonary membrane and the pulmonary capillary blood volume in cardiopulmonary disease. Clin. Sci. 1958, 17, 465.

8. Donevan, R. E., Palmer, W. H., Varvis, C. J., and Bates, D. V. Influence of age on pulmonary diffusing capacity. J. appl. Physiol. 1959, 14, 483.

9. Lawther, P. J., and Apthorp, G. H. A method for the determination of carbon monoxide in blood. Brit. J. industr. Med. 1955, 12, 326.

10. Linderholm, $\mathrm{H}$. On the significance of $\mathrm{CO}$ tension in pulmonary capillary blood for determination of pulmonary diffusing capacity with the steady state CO method. Acta med. scand. 1957, 156, 413.

11. Asmussen, E., and Nielsen, M. Physiological dead space and alveolar gas pressures at rest and during muscular exercise. Acta physiol. scand. 1956, 38, 1.

12. Bishop, J. M., Donald, K. W., and Wade, O. L. Circulatory dynamics at rest and on exercise in the hyperkinetic states. Clin. Sci. 1955, 14, 329.

13. Cotes, J. E. The role of oxygen, carbon dioxide and lactic acid in the ventilatory response to exercise in patients with mitral stenosis. Clin. Sci. 1955, $14,317$.

14. MacIntosh, D. J., Sinnott, J. C., Milne, I. G., and Reid, E. A. Some aspects of disordered pulmonary function in mitral stenosis. Ann. intern. Med. 1958, 49, 1294.

15. Riley, R. L., Riley, M. C., and Hill, H. McD. Diffuse pulmonary sarcoidosis. Johns Hopk. Hosp. Bull. 1952, 91, 345.

16. Marshall, R., Smellie, H., Baylis, J. H., Hoyle, C., and Bates, D. V. Pulmonary function in sarcoidosis. Thorax 1958, 13, 48.

17. Ross, J. C., Frayser, R., and Hickam, J. B. A study of the mechanism by which exercise increases the pulmonary diffusing capacity for carbon monoxide. J. clin. Invest. 1959, 38, 916.

18. Turino, G. M., Brandfonbrener, M., and Fishman, A. P. The effect of changes in ventilation and pulmonary blood flow on the diffusing capacity of the lung. J. clin. Invest. 1959, 38, 1186.

19. McIlroy, M. B., and Bates, D. V. Respiratory function after pneumonectomy. Thorax 1956, 11, 303.

20. Linderholm, $H$. Diffusing capacity of the lungs as a limiting factor for physical working capacity. Acta med. scand. 1959, 163, 61. 

21. Auchincloss, J. H., Jr., Gilbert, R., and Eich, R. H.
The pulmonary diffusing capacity in congenital and rheumatic heart disease. Circulation 1959, 19, 232.

22. Rosenberg, E., and Forster, R. E. Relative importance of intravascular pressure and blood flow in determining the diffusing capacity of isolated perfused cat lungs. Fed. Proc. 1959, 18, 130.
23. Riley, R. L., Johns, C. J., Cohen, G., Cohn, J. E., Carroll, D. G., and Shepard, R. H. The diffusing capacity of the lungs in patients with mitral stenosis studied post-operatively. J. clin. Invest. 1956, 35, 1008.

24. Bink, B. The physical working capacity of cardiac patients in Monograph from Netherlands Institute for Preventive Medicine. Leyden, Holland, 1959. 